\title{
Undecidability of Context-Sensitive Data-Dependence Analysis
}

\author{
THOMAS REPS \\ University of Wisconsin
}

\begin{abstract}
A number of program-analysis problems can be tackled by transforming them into certain kinds of graph-reachability problems in labeled directed graphs. The edge labels can be used to filter out paths that are not of interest: A path $P$ from vertex $s$ to vertex $t$ only counts as a "valid connection" between $s$ and $t$ if the word spelled out by $P$ is in a certain language. Often the languages used for such filtering purposes are languages of matching parentheses:

- In some cases, the matched-parenthesis condition is used to filter out paths with mismatched calls and returns. This leads to so-called "context-sensitive" program analyses, such as context-sensitive interprocedural slicing and contextsensitive interprocedural dataflow analysis.

- In other cases, the matched-parenthesis condition is used to capture a graph-theoretic analog of McCarthy's rules: "car $(\operatorname{cons}(x, y))=x "$ and " $\operatorname{cdr}(\operatorname{cons}(x, y))=y$ ". That is, in the code fragment

$$
\begin{aligned}
& c=\operatorname{cons}(a, b) ; \\
& d=\operatorname{car}(c) ;
\end{aligned}
$$
\end{abstract}

the fact that there is a "structure-transmitted data dependence" from a to $d$, but not from b to d, is captured in a graph by using (i) a vertex for each variable, (ii) an edge from vertex $i$ to vertex $j$ when $i$ is used on the right-hand side of an assignment to $j$, (iii) parentheses that match as the labels on the edges that run from a to $\mathrm{c}$ and $\mathrm{c}$ to $\mathrm{d}$, and (iv) parentheses that do not match as the labels on the edges that run from $\mathrm{b}$ to $\mathrm{c}$ and $\mathrm{c}$ to $\mathrm{d}$.

However, structure-transmitted data-dependence analysis is context-insensitive, because there are no constraints that filter out paths with mismatched calls and returns. Thus, a natural question is whether these two kinds of uses of parentheses can be combined to create a context-sensitive analysis for structure-transmitted data dependences. This paper answers the question in the negative: In general, the problem of context-sensitive, structure-transmitted data-dependence analysis is undecidable.

The results of this paper imply that, in general, both context-sensitive set-based analysis and $\infty$-CFA (when data constructors and selectors are taken into account) are also undecidable.

CR Categories and Subject Descriptors: D.3.4 [Programming Languages]: Processors—compilers; optimization; F.4.1 [Mathematical Logic and Formal Languages]: Mathematical Logic_computability theory; F.4.3 [Mathematical Logic and Formal Languages]: Formal Languages_decision problems; G.2.2 [Discrete Mathematics]: Graph Theory-path and circuit problems;

General Terms: Languages, Theory

Additional Key Words and Phrases: Context-sensitive program analysis, dependence analysis, graph-reachability problem, structure-transmitted data dependence, set-based analysis, set constraints, control-flow analysis, $\infty$-CFA, linear matched-parenthesis language

This work was supported in part by the National Science Foundation under grants CCR-9625667 and CCR-9619219, by the United States-Israel Binational Science Foundation under grant 96-00337, by a grant from IBM, and by a Vilas Associate Award from the University of Wisconsin.

The U.S. Government is authorized to reproduce and distribute reprints for Governmental purposes, notwithstanding any copyright notices affixed thereon. The views and conclusions contained herein are those of the authors, and should not be interpreted as necessarily representing the official policies or endorsements, either expressed or implied, of the above government agencies or the U.S. Government.

Author's address: Computer Sciences Department, University of Wisconsin, 1210 W. Dayton St., Madison, WI 53706.

E-mail: reps@cs.wisc.edu. 


\section{INTRODUCTION}

A number of program-analysis problems can be tackled by transforming them into certain kinds of graphreachability problems in labeled directed graphs $[20,9,5,4,6,15,19,28,30,16,29,25,32]$. It is useful to consider not just ordinary reachability (e.g., transitive closure), but a generalization in which the edge labels are used, in effect, to filter out paths that are not of interest [4,15,28,30,16,29,25,32]: A path $P$ from vertex $s$ to vertex $t$ only counts as a "valid connection" between $s$ and $t$ if the word spelled out by $P$ (i.e., the concatenation, in order, of the labels on the edges of $P$ ) is in a certain language.

Definition 1.1. Let $L$ be a language over alphabet $\Sigma_{L}$, and let $G$ be a graph whose edges are labeled with members of $\Sigma_{L}$. Each path in $G$ defines a word over $\Sigma_{L}$, namely, the word obtained by concatenating, in order, the labels of the edges on the path. A path in $G$ is an L-path if its word is a member of $L$. An instance of the (single-source/single-target) $L$-path problem asks whether there exists an $L$-path in $G$ from a given source vertex $s$ to a given target vertex $t$.

Let $\mathcal{L}$ be a family of languages, and $L \in \mathcal{L}$ be a language over alphabet $\Sigma_{L}$. An instance of the $\mathcal{L}$-reachability problem is an $L$-path problem instance $\left\langle L, \Sigma_{L}, G, s, t\right\rangle$.

Example. When the family of languages $\mathcal{L}$ in Defn. 1.1 is the context-free languages, we have the $C F L$ reachability problem [38]. Consider the graph and the context-free grammar shown below. Note that $L$ (matched) is the context-free language that consists of strings of matched parentheses and square brackets, with zero or more $e$ 's interspersed. ${ }^{1}$
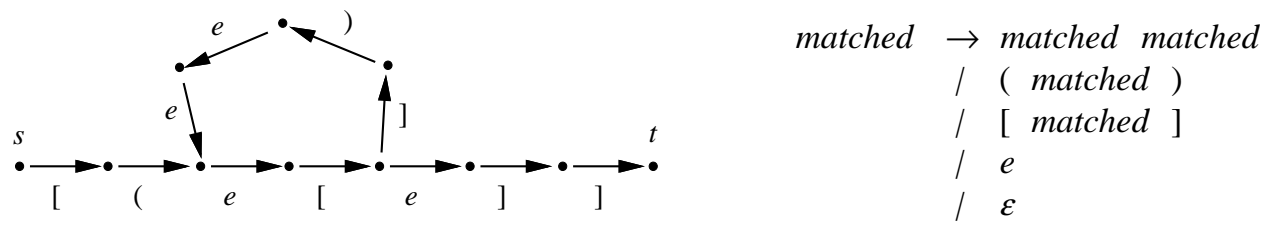

In this graph, there is exactly one $L$ (matched)-path from $s$ to $t$ : The path goes exactly once around the cycle, and generates the word " $[(e[]) e e e[e]]$ ".

A number of program-analysis problems can be viewed as instances of the CFL-reachability problem [32]. In program-analysis problems, the languages used for such filtering purposes are often languages of matching parentheses. In some cases, the matched-parenthesis condition is used to filter out paths with mismatched calls and returns in order to implement so-called "context-sensitive" program analyses.

Example 1.2. The use of CFL-reachability in context-sensitive program analysis, as opposed to ordinary graph reachability, is illustrated by the following example: ${ }^{2}$

\footnotetext{
${ }^{1}$ In this paper, the word "parentheses" is used in both the generic sense- to mean any kind of matching delimiter (e.g., round parentheses, square brackets, curly braces, angle brackets, etc.) - as well as in the specific sense of round parentheses. It should always be clear from the context which of these two meanings is intended.

${ }^{2}$ In this example, we use $\mathrm{C}$ syntax. In later examples, we use $\mathrm{C}$ augmented with the operator cons, which denotes a pairing constructor; the operators car and cdr, which select the first and second components of a pair, respectively; and the operator atom, which constructs an atomic object (different from NULL) from a given string. This notation is used to simplify the way storage-allocation operations are expressed in our examples. The use of these operators does not imply that our results apply only to the analysis of LISP programs.
} 

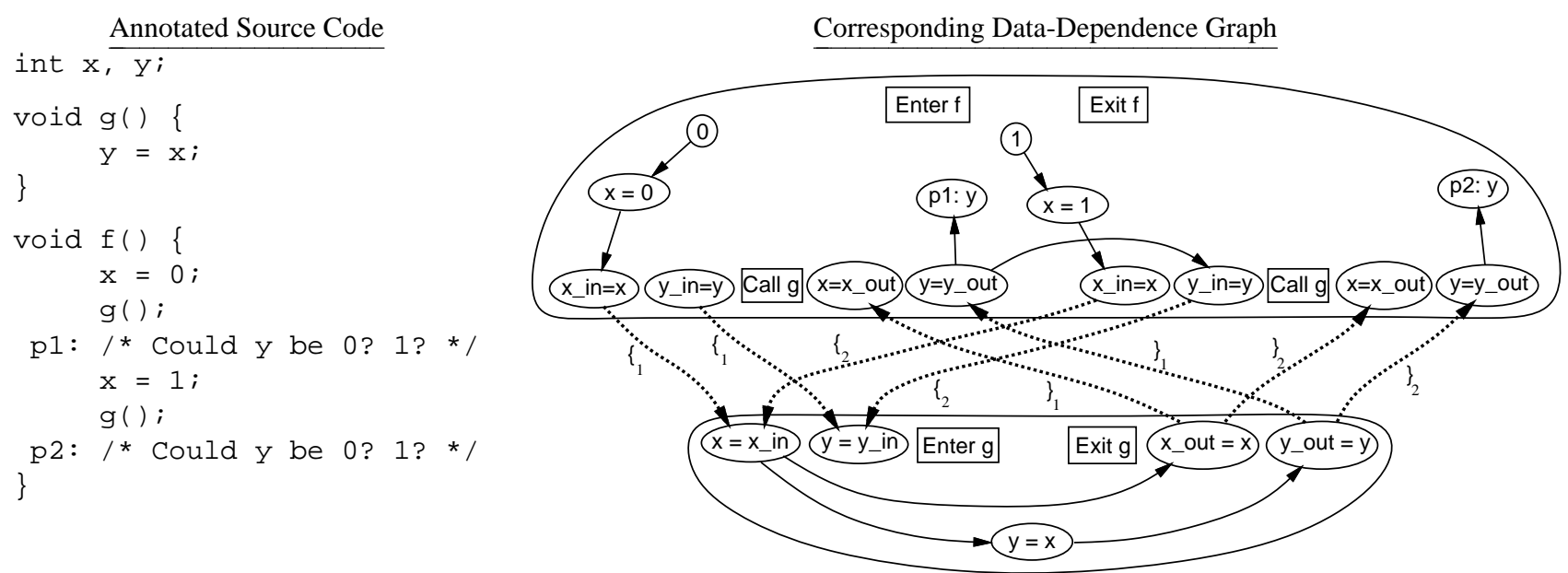

The diagram on the right shows the program's data-dependence graph. (Strictly speaking, neither the two large ovoid shapes nor the rectangular boxes labeled Call, Enter, and Exit are part of the data-dependence graph. The two ovoids indicate which elements belong to which procedure; the rectangular boxes provide some context about the control points in the program to which the various vertices are associated.) Each directed edge in the graph represents a data dependence (also known as a flow dependence [21,22]): An edge from vertex $v_{1}$ to vertex $v_{2}$ indicates that the value produced at $v_{1}$ may be used at vertex $v_{2}$. For instance, the edge

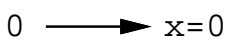

in the dependence graph for procedure $f$ indicates that the value of $x$ after the execution of the statement $\mathrm{x}=0$ could be (and, in this case, must be) 0 .

In the above program, procedure $g$ has no parameters. However, our data-dependence graphs reflect a somewhat nonstandard treatment of global variables: A global variable such as $\mathrm{x}$ is treated as if it were a "hidden" value-result parameter whose value (and subsequent return value) is passed from one scope to another via the special scope-transfer variables $x_{\text {z }}$ in and $x_{\text {z }}$ out. For example, the interprocedural datadependence edge

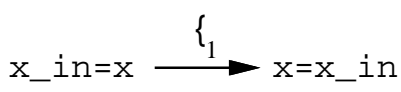

represents the passing of $x$ from $f$ 's scope to g's scope at the first call on $g$. Note that data-dependence edges for dependences transmitted from the caller to the callee (i.e., from $f$ to $g$ ) are labeled by the symbols " $\left\{\begin{array}{l}1 \\ 1\end{array}\right.$ and " $\left\{_{2}\right.$ ", whereas data-dependence edges for dependences transmitted from the callee back to the caller are labeled by the symbols " $\}_{1}$ " and " $\}_{2}$ ". In particular, the data-dependence edges that represent how $\mathrm{x}$ and $\mathrm{y}$ are passed from $f$ 's scope to $g$ 's scope at the first call on $g$ are labeled with $\left\{{ }_{1}\right.$; the datadependence edges that represent how $\mathrm{x}$ and $\mathrm{y}$ are passed from f's scope to $g$ 's scope at the second call on $g$ are labeled with $\left\{_{2}\right.$. Likewise, the data-dependence edges that represent how $\mathrm{x}$ and $\mathrm{y}$ are passed back from g's scope to $f$ 's scope after the two calls finish are labeled with $\}_{1}$ and $\}_{2}$, respectively.

Our data-dependence graphs also have vertices that correspond to various annotations in the program; annotations, denoted here as comments, indicate that we are interested in a given variable at a given point in the program. In the example above, the comments at $\mathrm{p} 1$ and $\mathrm{p} 2$ give rise to the vertices $\mathrm{p} 1: \mathrm{y}$ and $\mathrm{p} 2: \mathrm{y}$.

A context-insensitive analysis that tracks dependences between constants and variables in the program will report that $\mathrm{y}$ depends on 0 and 1 at both $\mathrm{p} 1$ and $\mathrm{p} 2$. The reason is that a context-insensitive analysis ignores the fact that the only paths that can possibly be feasible execution paths are those in which returns are matched with corresponding calls. For instance, the existence of the (mismatched) path 


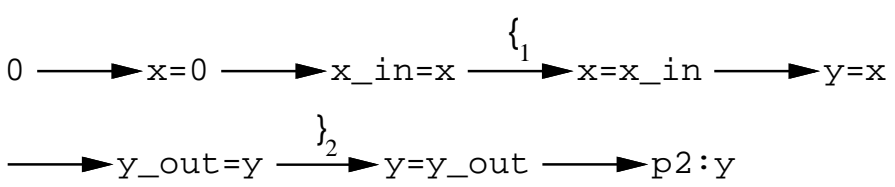

in the graph shown above serves as "evidence" that $\mathrm{p} 2: \mathrm{y}$ depends on 0 (i.e., that the value of $\mathrm{y}$ at $\mathrm{p} 2$ could be 0 ).

In contrast, a context-sensitive analysis only reports possible transmissions of values along paths in which returns are matched with corresponding calls. For this example, it reports that $\mathrm{p} 1: \mathrm{y}$ depends on 0 but not on 1 and that $\mathrm{p} 2$ : y depends on 1 but not on 0 (i.e., y could have only the value 0 at $\mathrm{p} 1$ and 1 at $\mathrm{p} 2$ ). The context-sensitive analysis can be expressed as a CFL-reachability problem with respect to a language of matched indexed curly braces. It would say that path (1.3) is not a valid connection between 0 and $\mathrm{p} 2: \mathrm{y}$, because the label $\left\{_{1}\right.$ on the interprocedural data-dependence edge $\mathrm{x}_{-}$in $=\mathrm{x} \stackrel{\left\{_{1}\right.}{\longrightarrow} \mathrm{x}=\mathrm{x}$ _in (which represents the transfer of $x$ 's value from $f$ to $g$ as $g$ is entered at the first call site) does not match the label $\}_{2}$ on the edge y_out $=\mathrm{y} \stackrel{\}_{2}}{\longrightarrow}$ y=y_out (which represents the transfer of y's value back to $f$ when a return is performed in $g$ from a call on $g$ that originated at the second call site). On the other hand, because $\left\{{ }_{2} \text { matches with }\right\}_{2}$, the (matched) path

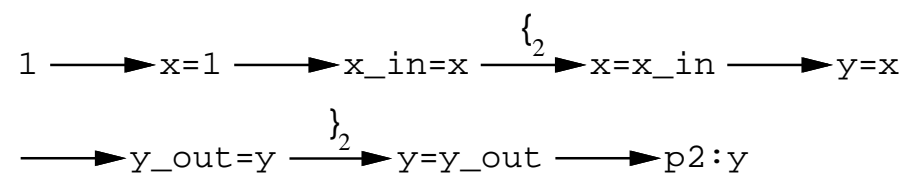

does count as a valid connection between 1 and $\mathrm{p} 2: \mathrm{y}$, and this path serves as evidence that the value of $\mathrm{y}$ at $\mathrm{p} 2$ could be 1 .

Problems in which CFL-reachability has been used to devise context-sensitive program analyses include interprocedural slicing $[15,28]$ and interprocedural dataflow analysis $[30,16] .^{3}$

CFL-reachability - and, in particular, a matched-parenthesis constraint-has also been used to capture a graph-theoretic analog [29,25] of McCarthy's rules [24] (i.e., “car (cons $(x, y))=x$ " and "cdr ( cons $(x, y))=y ")$, as illustrated in the following example.

Example 1.5. The following program illustrates the use of CFL-reachability in (context-insensitive) structure-transmitted data-dependence analysis:

\footnotetext{
${ }^{3}$ There is an unfortunate clash in terminology that the reader should be aware of. The term "context-sensitive analysis" is standard in the programming-languages community, where it means a static-analysis method in which the analysis of a called procedure is "sensitive" to the context in which it is called: A context-sensitive analysis captures the fact that different call sites that call the same procedure may have different effects on a program's possible execution states. Context-sensitive analysis should not be confused with the "context-sensitive languages" of formal-language theory. Unfortunately, the principle that context-free-language reachability is useful in formalizing approaches to context-sensitive analysis was fully articulated [32] only after the term "context-sensitive analysis" had been adopted by the programming-languages community [7,37].
} 

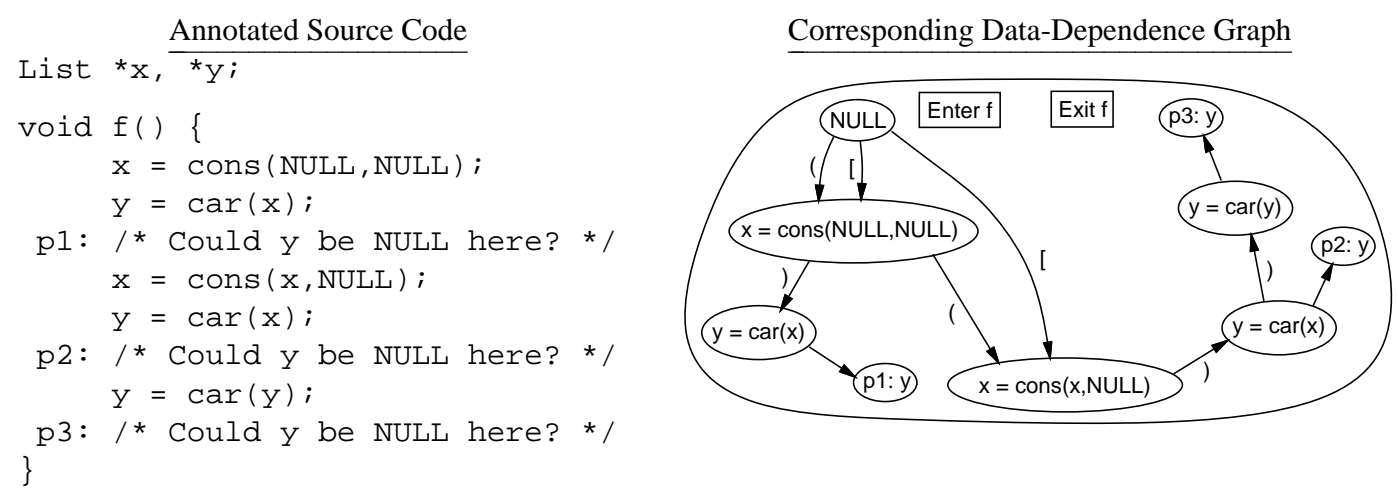

In this graph, the labels on the data-dependence edges serve a different purpose than the labels used in Example 1.2: Here an edge labeled "(" corresponds to a data construction in which the value is placed in the first position of a cons; an edge labeled "[" corresponds to a data construction in which the value is placed in the second position of a cons; an edge labeled ")" corresponds to a selection via car; an edge labeled "]" corresponds to a selection via cdr.

The matched-parenthesis path

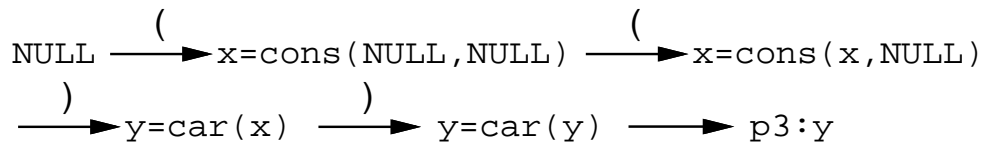

from NULL to $\mathrm{p} 3: \mathrm{y}$ serves as evidence that the value of $\mathrm{y}$ at $\mathrm{p} 3$ could be NULL. The path

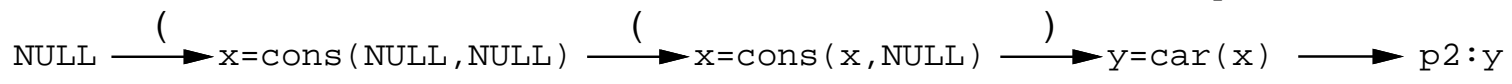

does not serve as evidence that the value of y at p2 could be NULL, because the first occurrence of "(" has no matching ")". In fact, there is no matched-parenthesis path from NULL to p2:y, and a (context-insensitive) structure-transmitted data-dependence analysis would conclude that the value of y at p2 cannot be NULL. $\square$

The structure-transmitted data-dependence analyses given in [29] and [25] are context-insensitive, because there are no constraints that filter out paths with mismatched calls and returns. Thus, a natural question is whether the two kinds of uses of matching delimiters illustrated in Examples 1.2 and 1.5 can be combined to create a context-sensitive analysis for structure-transmitted data dependences. The following interprocedural variation on Example 1.5 illustrates what we would hope to gain from a context-sensitive, structure-transmitted data-dependence analysis:

Example 1.6. Consider the following example program: 


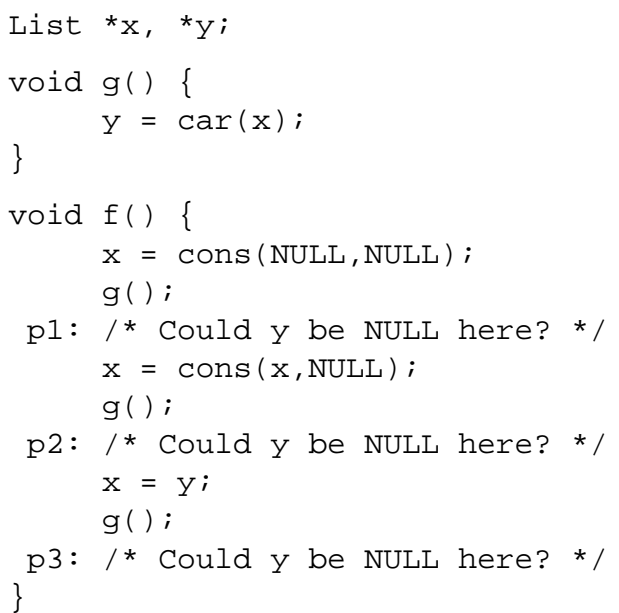

The relevant portions of this program's data-dependence graph are shown below:

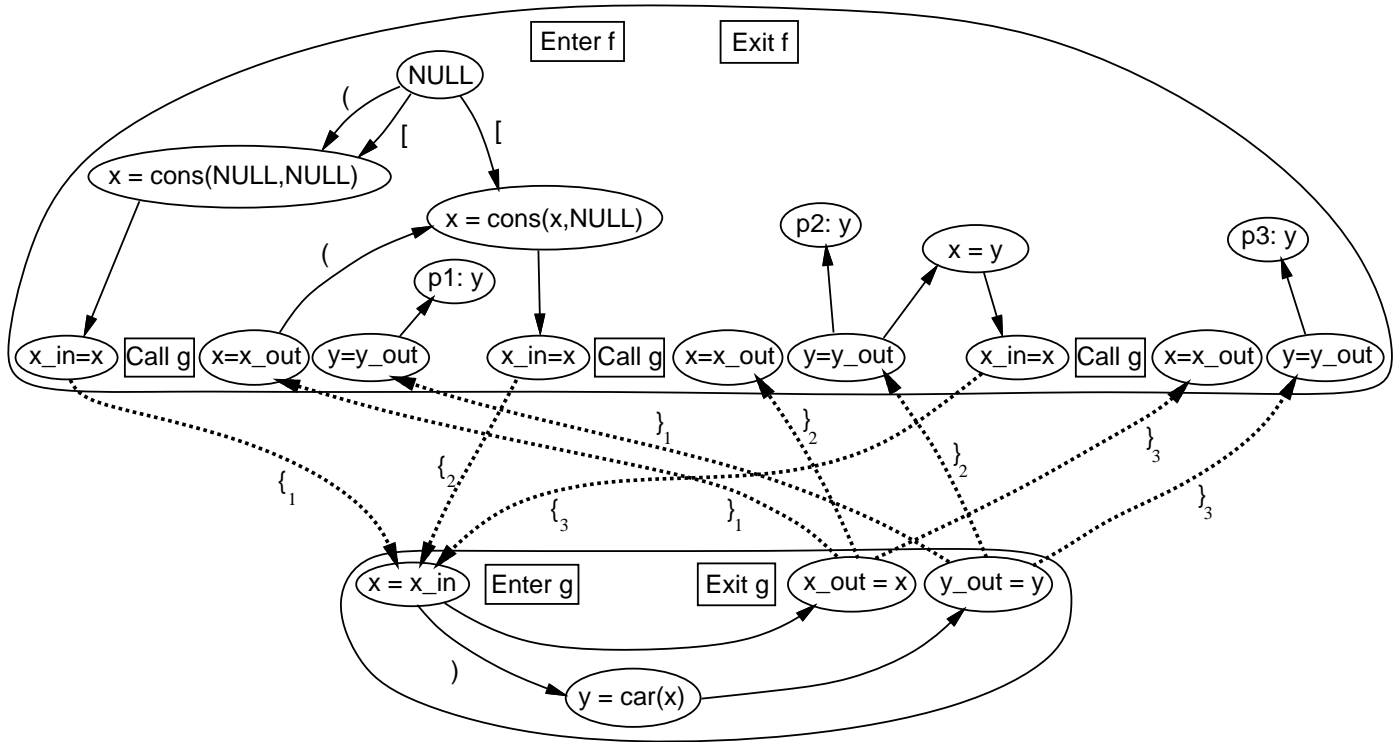

For this example, a context-sensitive analysis should report that variable y can have the value NULL at $\mathrm{p} 1$ and $\mathrm{p} 3$, but not at $\mathrm{p} 2$. The following path from NULL to program point $\mathrm{p} 3$ serves as evidence that the value of $y$ at $p 3$ could be NULL:

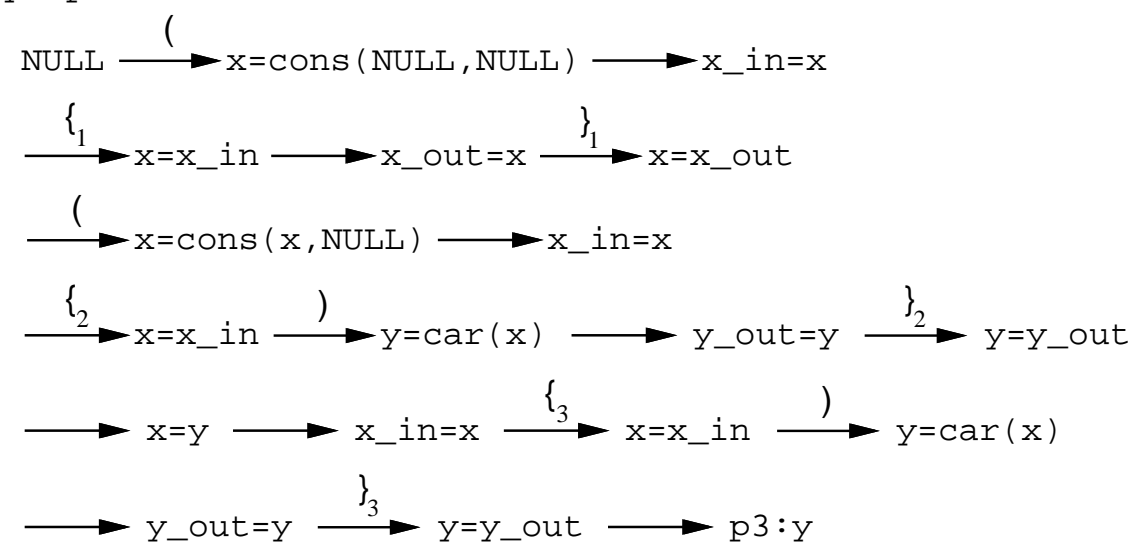


In contrast, a context-insensitive analysis would also use the following path from NULL to program point $\mathrm{p} 2$ as evidence that the value of $\mathrm{y}$ at $\mathrm{p} 2$ could be NULL:

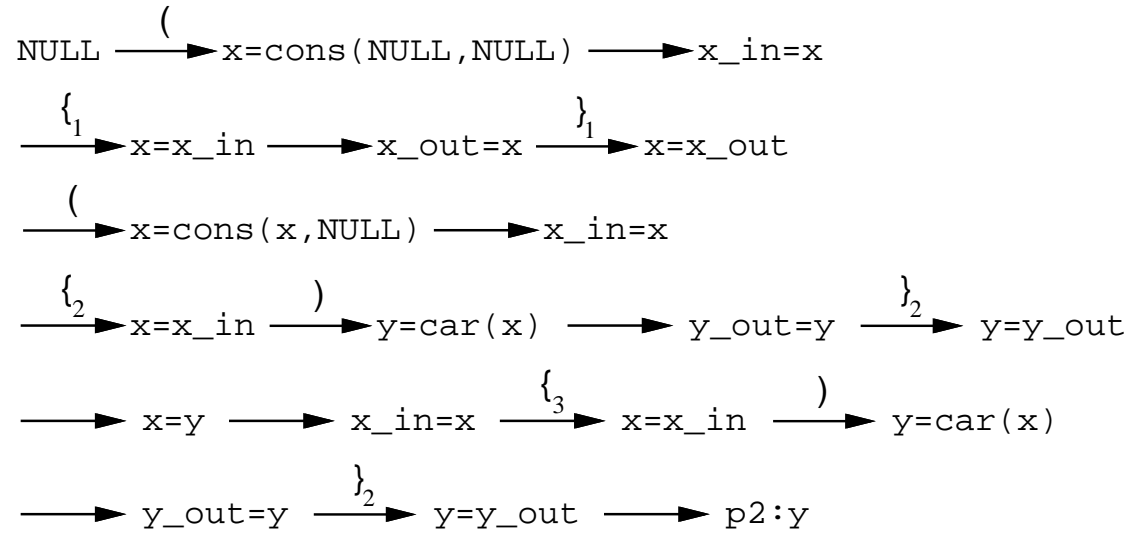

The problem with this path is that there is a mismatch between the labels on the edge $\mathrm{x}$ _in $=\mathrm{x} \stackrel{\left\{_{3}\right.}{\longrightarrow} \mathrm{x}=\mathrm{x}$ _in and the subsequent edge $\mathrm{y}$ _out $=\mathrm{y} \stackrel{\}_{2}}{\longrightarrow} \mathrm{y}=\mathrm{y}$ _out. Consequently, this path would be excluded from consideration by a context-sensitive analysis.

The other fact to note about Example 1.6 is that in path (1.7), although "(" symbols match with ")" symbols, and " $\left\{{ }_{i} \text { " symbols match with " }\right\}_{i}$ " symbols, the two patterns of matched symbols are interleaved. ${ }^{4}$ This observation serves to motivate the study of interleaved matched-parenthesis languages carried out in Sections 2 and 3.

This paper shows that it is impossible to create an algorithm that captures all, and only, interleaved matched-parenthesis paths of the kind illustrated in Example 1.6: In general, the problem of context-sensitive, structure-transmitted data-dependence analysis is undecidable. In other words, you can capture either (i) the matching of calls and returns, or (ii) "car ( cons $(x, y))=x$ cancellation", but not both simultaneously, in any amount of time. (Of course, there may be useful algorithms that compute approximate, but safe, solutions to this problem, $c f$. [13].)

In terms of the programming-language features needed for this result to apply, higher-order functions are not required: The main result of this paper implies that context-sensitive, structure-transmitted data-dependence analysis is undecidable for first-order languages (both functional and imperative). This result applies to such languages as C, C++, Java, ML, and Scheme, as well as to many others.

It should be noted that questions of the kind posed in Example 1.6 (i.e., "Does a given variable have a given value at a particular point in a program?") often turn out to be undecidable in their most general form, and often there are several independent reasons why the problem is undecidable (e.g., it is undecidable whether a given statement is ever executed; it is undecidable whether a given path is ever executed; etc.). This paper shows that context-sensitive, structure-transmitted data-dependence analysis is undecidable even if a conservative approximation is made that, for many other program-analysis problems, overcomes other sources of undecidability. (In particular, we assume that all paths in a procedure's control-flow graph are executable.)

The remainder of the paper is organized into four sections: The undecidability result is shown by a reduction from a variant of Post's Correspondence Problem (PCP); Section 2 defines PCP and discusses a variant of it that is particularly suited to our needs. Example 1.6 motivates our interest in languages with interleaved patterns of matching delimiters; Section 3 shows that a certain set of $L$-path problem instanceswhere $L$ is a language of strings formed by interleaving two languages of matching parentheses-is unde-

${ }^{4}$ In this paper, the term "interleaved" is used in a somewhat restricted sense, compared to the standard usage in formal-language theory (cf. [14, pp. 282]). The exact nature in which patterns of matched symbols are allowed to be woven together is defined precisely in Sections 3 and 4 . 
cidable. Section 4 relates the result from Section 3 to the undecidability of context-sensitive, structuretransmitted data-dependence analysis. Section 5 discusses what our results imply about other programanalysis problems.

\section{A VARIANT OF POST'S CORRESPONDENCE PROBLEM}

Our undecidability result is shown by a reduction from a variant of Post's Correspondence Problem:

Definition 2.1. An instance of Post's Correspondence Problem, or PCP, consists of two lists of strings, $X$ and $Y$, where $X$ and $Y$ each consist of $k$ strings in $\{0,1\}^{+}$:

$$
\begin{aligned}
& X=x_{1}, x_{2}, \cdots, x_{k} \\
& Y=y_{1}, y_{2}, \cdots, y_{k}
\end{aligned}
$$

The instance of PCP has a solution if there exists a nonempty sequence of integers $i_{1}, i_{2}, \cdots, i_{j}, \cdots, i_{m}$ such that (i) for all $1 \leq j \leq m$, we have $1 \leq i_{j} \leq k$, and (ii) $x_{i_{1}} x_{i_{2}} \cdots x_{i_{j}} \cdots x_{i_{m}}=y_{i_{1}} y_{i_{2}} \cdots y_{i_{j}} \cdots y_{i_{m}}$.

Example 2.2. Consider the following instance of PCP, where $k$ is 3 :

$$
\begin{aligned}
& X=0101,101,111 \\
& Y=01, \quad 011,0111101
\end{aligned}
$$

This instance of PCP has the solution 1,2,3,1 because

$$
x_{1} x_{2} x_{3} x_{1}=01011011110101=01011011110101=y_{1} y_{2} y_{3} y_{1} \text {. }
$$

PCP is known to be undecidable; for proofs, see Hopcroft and Ullman [14, pp. 193-198], Lewis and Papadimitriou [23, pp. 289-293], or Harrison [8, pp. 249-256].

For our purposes, it is more convenient to work with the following variant of PCP:

Definition 2.3. (Parenthesis-PCP) Given an instance of PCP,

$$
\begin{aligned}
& X=x_{1}, x_{2}, \cdots, x_{k} \\
& Y=y_{1}, y_{2}, \cdots, y_{k}
\end{aligned}
$$

we define the corresponding instance of parenthesis-PCP, or P-PCP, as

$$
\begin{aligned}
\bar{X} & =\bar{x}_{1}, \bar{x}_{2}, \cdots, \bar{x}_{k} \\
\bar{Y}^{R} & =\bar{y}_{1}^{R}, \bar{y}_{2}^{R}, \cdots, \bar{y}_{k}^{R}
\end{aligned}
$$

where, for $1 \leq i \leq k$,

- $\bar{x}_{i}$ is the string in $\left\{\left(,[\}^{+}\right.\right.$equal to $x_{i}$ with 0 replaced by "(" and 1 replaced by "[".

- $\bar{y}_{i}$ is the string in $\{$ ), $\left.]\right\}^{+}$equal to $y_{i}$ with 0 replaced by “)" and 1 replaced by "]".

- The superscript " $R$ " on a string denotes the reversed string.

A solution to an instance of P-PCP is defined with the aid of the following linear context-free grammar: ${ }^{5}$

$$
\begin{aligned}
& \text { balanced } \rightarrow \text { ( balanced ) } \\
& \text { [ [ balanced ] } \\
& \text { ( \#) } \\
& \text { | [ \# ] }
\end{aligned}
$$

An instance of P-PCP has a solution if there exists a nonempty sequence of integers

$i_{1}, \quad i_{2}, \quad \cdots, \quad i_{j}, \quad \cdots, \quad i_{m}$ such that (i) for all $1 \leq j \leq m$, we have $1 \leq i_{j} \leq k$, and (ii) $\bar{x}_{i_{1}} \bar{x}_{i_{2}} \cdots \bar{x}_{i_{j}} \cdots \bar{x}_{i_{m}} \# \bar{y}_{i_{m}}^{R} \cdots \bar{y}_{i_{j}}^{R} \cdots \bar{y}_{i_{2}}^{R} \bar{y}_{i_{1}}^{R} \in L($ balanced $)$.

Example 2.4. The instance of P-PCP that corresponds to Example 2.2 is

${ }^{5} \mathrm{~A}$ linear context-free grammar is one in which at most one nonterminal appears on the right-hand side of each production. 


$$
\begin{aligned}
& \bar{X}=([([, \quad[([, \quad[[[] \\
& \left.\bar{Y}^{R}=\text { ]), ]] }\right), \text { ])]]]]) }
\end{aligned}
$$

This instance of P-PCP has the solution 1, 2, 3, 1 because

$$
\left.\bar{x}_{1} \bar{x}_{2} \bar{x}_{3} \bar{x}_{1} \# \bar{y}_{1}^{R} \bar{y}_{3}^{R} \bar{y}_{2}^{R} \bar{y}_{1}^{R}=([([[([[[([([\#)])]]]])]])]) \in \text { L(balanced }\right) .
$$

Clearly an instance of PCP has a solution if and only if the corresponding instance of P-PCP has a solution.

For a given instance of P-PCP,

$$
\begin{aligned}
\bar{X} & =\bar{x}_{1}, \bar{x}_{2}, \cdots, \bar{x}_{k} \\
\bar{Y}^{R} & =\bar{y}_{1}^{R}, \bar{y}_{2}^{R}, \cdots, \bar{y}_{k}^{R}
\end{aligned}
$$

every solution (if one exists) corresponds to a string in the language generated by the following linear context-free grammar:

$$
\begin{aligned}
& S_{0} \rightarrow \bar{x}_{i} S_{0} \quad \bar{y}_{i}^{R} \quad \text { for } 1 \leq i \leq k \\
& \text { | } \bar{x}_{i} \# \bar{y}_{i}^{R} \quad \text { for } 1 \leq i \leq k
\end{aligned}
$$

While not every string in $L\left(S_{0}\right)$ corresponds to a solution, all strings in $L\left(S_{0}\right)$ that are also in $L($ balanced) correspond to a solution. That is, the given instance of P-PCP has a solution exactly when the language $L\left(S_{0}\right) \cap L($ balanced $)$ is non-empty. This observation implies the following theorem (cf. [23, pp. 293-294]):

THEOREM 2.5. It is undecidable for arbitrary linear context-free grammars $G_{1}$ and $G_{2}$ whether $L\left(G_{1}\right) \cap L\left(G_{2}\right)$ is empty.

The fact that the existence of a solution to a given instance of P-PCP can be characterized by the nonemptiness of the intersection of two linear context-free grammars underlies our result on the undecidability of context-sensitive data-dependence analysis. However, for the purpose of investigating the latter problem, it is useful to develop a slightly more elaborate way of characterizing the solutions to an instance of P-PCP:

Definition 2.6. If an instance of P-PCP

$$
\begin{aligned}
\bar{X} & =\bar{x}_{1}, \bar{x}_{2}, \cdots, \bar{x}_{k} \\
\bar{Y}^{R} & =\bar{y}_{1}^{R}, \bar{y}_{2}^{R}, \cdots, \bar{y}_{k}^{R}
\end{aligned}
$$

has a solution $i_{1}, i_{2}, \cdots, i_{m}$, we say that the following string exhibits the solution in tagged form: ${ }^{6}$

$$
\left\{{ }_{i_{1}} \bar{x}_{i_{1}}\left\{{ }_{i_{2}} \bar{x}_{i_{2}} \cdots\left\{{ }_{i_{m}} \bar{x}_{i_{m}} \# \bar{y}_{i_{m}}^{R}\right\}_{i_{m}} \cdots \bar{y}_{i_{2}}^{R}\right\}_{i_{2}} \bar{y}_{i_{1}}^{R}\right\}_{i_{1}} \text {. }
$$

In general, suppose that $i_{1}, i_{2}, \cdots, i_{j}, \cdots, i_{p}$, is some nonempty sequence of integers such that, for all $1 \leq j \leq p$, we have $1 \leq i_{j} \leq k$. Regardless of whether $i_{1}, i_{2}, \cdots, i_{j}, \cdots, i_{p}$ is actually a solution to the instance of P-PCP, we say that a string of the form

$$
\left\{{ }_{i_{1}} \bar{x}_{i_{1}}\left\{{ }_{i_{2}} \bar{x}_{i_{2}} \cdots\left\{_{i_{j}} \bar{x}_{i_{j}} \cdots\left\{_{i_{p}} \bar{x}_{i_{p}} \# \bar{y}_{i_{p}}^{R}\right\}_{i_{p}} \cdots \bar{y}_{i_{j}}^{R}\right\}_{i_{j}} \cdots \bar{y}_{i_{2}}^{R}\right\}_{i_{2}} \bar{y}_{i_{1}}^{R}\right\}_{i_{1}}
$$

exhibits a candidate solution in tagged form.

For instance, because the sequence 2, 1, 2, 3, 1 is not a solution to Example 2.4, the following string exhibits a candidate solution in tagged form (but not a solution in tagged form):

$$
\left\{_{2}\left[\left(\left[\left\{_{1}\left(\left[\left(\left[\left\{_{2}\left[\left(\left[\left\{_{3}\left[\left[\left[\left\{_{1}\left([([\#])\}_{1}\right]\right)\right]\right]\right]\right]\right)\right\}_{3}\right]\right]\right)\right\}_{2}\right]\right)\right\}_{1}\right]\right]\right)\right\}_{2} \text {. }
$$

In contrast, the following string exhibits a candidate solution to Example 2.4 in tagged form that is also a solution in tagged form:

${ }^{6}$ For technical reasons having to do with the details of the constructions given in Sections 3 and 4, we will also work with some slight variants of (2.7). For instance, in Section 3 we use a version of (2.7) in which each occurrence of " $\{$ " is immediately preceded by two occurrences of the symbol $e$, and each occurrence of " \}" is immediately followed by two occurrences of $e$. 


$$
\left\{_{1}\left(\left[\left(\left[\left\{_{2}\left[\left(\left[\left\{_{3}\left[\left[\left[\left\{_{1}\left([([\#])\}_{1}\right]\right)\right]\right]\right]\right]\right)\right\}_{3}\right]\right]\right)\right\}_{2}\right]\right)\right\}_{1} .
$$

\section{AN UNDECIDABLE FAMILY OF L-PATH PROBLEM INSTANCES}

In this section, we show how to formulate P-PCP in graph-theoretic terms. In particular, we construct an undecidable family of $L$-path problem instances, where each problem instance corresponds to an instance of P-PCP. Throughout the remainder of the paper, we assume that we have been given a fixed, but arbitrary, instance of P-PCP consisting of the $k$ pairs of strings

$$
\begin{aligned}
\bar{X} & =\bar{x}_{1}, \bar{x}_{2}, \cdots, \bar{x}_{k} \\
\bar{Y}^{R} & =\bar{y}_{1}^{R}, \bar{y}_{2}^{R}, \cdots, \bar{y}_{k}^{R}
\end{aligned}
$$

Our interest in P-PCP is motivated by the fact that a string that exhibits a P-PCP solution in tagged form has two interleaved patterns of matched delimiters:

(i) The string $\bar{x}_{i_{1}} \bar{x}_{i_{2}} \cdots \bar{x}_{i_{j}} \cdots \bar{x}_{i_{m}} \# \bar{y}_{i_{m}}^{R} \cdots \bar{y}_{i_{j}}^{R} \cdots \bar{y}_{i_{2}}^{R} \bar{y}_{i_{1}}^{R}$ is in L(balanced).

(ii) The string $\left\{_{i_{1}}\left\{_{i_{2}} \cdots\left\{i_{i_{j}} \cdots\left\{_{i_{m}} \#\right\}_{i_{m}} \cdots\right\}_{i_{j}} \cdots\right\}_{i_{2}}\right\}_{i_{1}}$ is in L(balanced'), the language of balanced strings made up of $\left\{{ }_{i} \text { and }\right\}_{i}$, for $1 \leq i \leq k$, defined by the following linear context-free grammar:

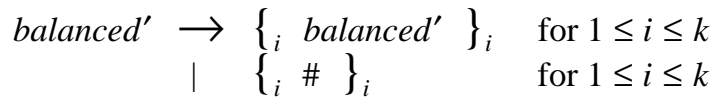

It is important to note that, in general, in a string that exhibits a P-PCP solution in tagged form, the two balancing processes can be out of sync. For instance, in the following prefix of string (2.9)

$$
\left\{_ { 1 } \left(\left[\left(\left[\left\{_ { 2 } \left[\left(\left[\left\{_ { 3 } \left[\left[\left[\left\{_ { 1 } \left([([\#])\}_{1}\right.\right.\right.\right.\right.\right.\right.\right.\right.\right.\right.\right.\right.\right.\right.
$$

the last symbol, namely " $\}_{1}$ ", does not match with the seventh-from-last symbol, namely "[". However, we can capture the structure of P-PCP solutions in tagged form via the intersection of two linear context-free languages:

(i) The language $L\left(S_{1}\right)$ consists of strings of $L$ (balanced) with an arbitrary number of symbols of the form " $\{$ " interspersed among the open-parenthesis and open-bracket symbols, and an arbitrary number of symbols of the form " $\}_{i}$ " interspersed among the close-parenthesis and close-bracket symbols:

$$
\begin{array}{lll}
S_{1} \rightarrow & \left(S_{1}\right) & \\
& {\left[S_{1}\right]} & \\
(\#) & \\
& {[\#]} & \\
& \left\{{ }_{i} S_{1}\right. & \text { for } 1 \leq i \leq k \\
& \left.S_{1}\right\}_{i} & \text { for } 1 \leq i \leq k
\end{array}
$$

(ii) The language $L\left(S_{2}\right)$ consists of all possible candidate solutions, in tagged form, to the given instance of P-PCP:

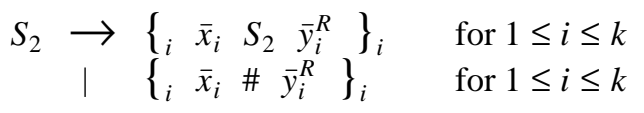

Languages $L\left(S_{1}\right)$ and $L\left(S_{2}\right)$ capture the two interleaved patterns of matched delimiters noted above: $L\left(S_{2}\right)$ consists of all possible candidate solutions, in tagged form, to the given instance of P-PCP; $L\left(S_{1}\right)$ consists of strings such that when all " $\left\{{ }_{i} \text { " and " }\right\}_{i}$ " symbols are excluded, we are left with a string in $L$ (balanced). Furthermore, the language $L\left(S_{1}\right) \cap L\left(S_{2}\right)$ consists of exactly the solutions, in tagged form, to the given instance of P-PCP. For instance, for Example 2.4, strings (2.8) and (2.9) are both in $L\left(S_{2}\right)$, but only string (2.9) is in $L\left(S_{1}\right)$; that is,

$$
\left\{_{1}\left(\left[\left(\left[\left\{_{2}\left[\left(\left[\left\{_{3}\left[\left[\left[\left\{_{1}\left([([\#])\}_{1}\right]\right)\right]\right]\right]\right]\right)\right\}_{3}\right]\right]\right)\right\}_{2}\right]\right)\right\}_{1} \in L\left(S_{1}\right) \cap L\left(S_{2}\right) .
$$

Similar to what we observed for the language $L\left(S_{0}\right) \cap L$ (balanced), the given instance of P-PCP has a solution exactly when the language $L\left(S_{1}\right) \cap L\left(S_{2}\right)$ is non-empty. 
We now show how to construct a graph (with two distinguished vertices, $s$ and $t$ ) such that there is an $L\left(S_{1}\right) \cap L\left(S_{2}\right)$-path from $s$ to $t$ if and only if the given instance of P-PCP has a solution. Fig. 1 shows a schematic diagram that illustrates the construction. For an instance of P-PCP $\bar{X}=\bar{x}_{1}, \bar{x}_{2}, \cdots, \bar{x}_{j}, \cdots, \bar{x}_{k}$, $\bar{Y}^{R}=\bar{y}_{1}^{R}, \bar{y}_{2}^{R}, \cdots, \bar{y}_{j}^{R}, \cdots, \bar{y}_{k}^{R}$, the graph contains $k$ regions of the form

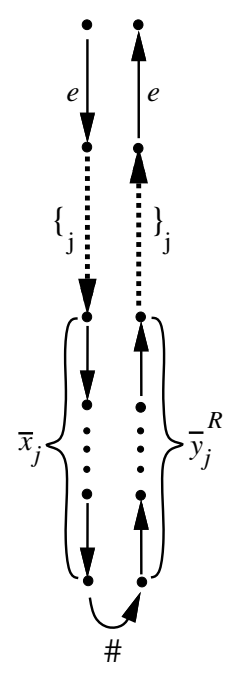

Call the left part of such a region an $x$-string segment, and the right part a $\bar{y}^{R}$-string segment. Note that the $j^{\text {th }} \bar{x}$-string segment begins with the sequence " $e\left\{{ }_{j}\right.$ ", whereas the $j^{\text {th }} \bar{y}^{R}$-string segment ends with the

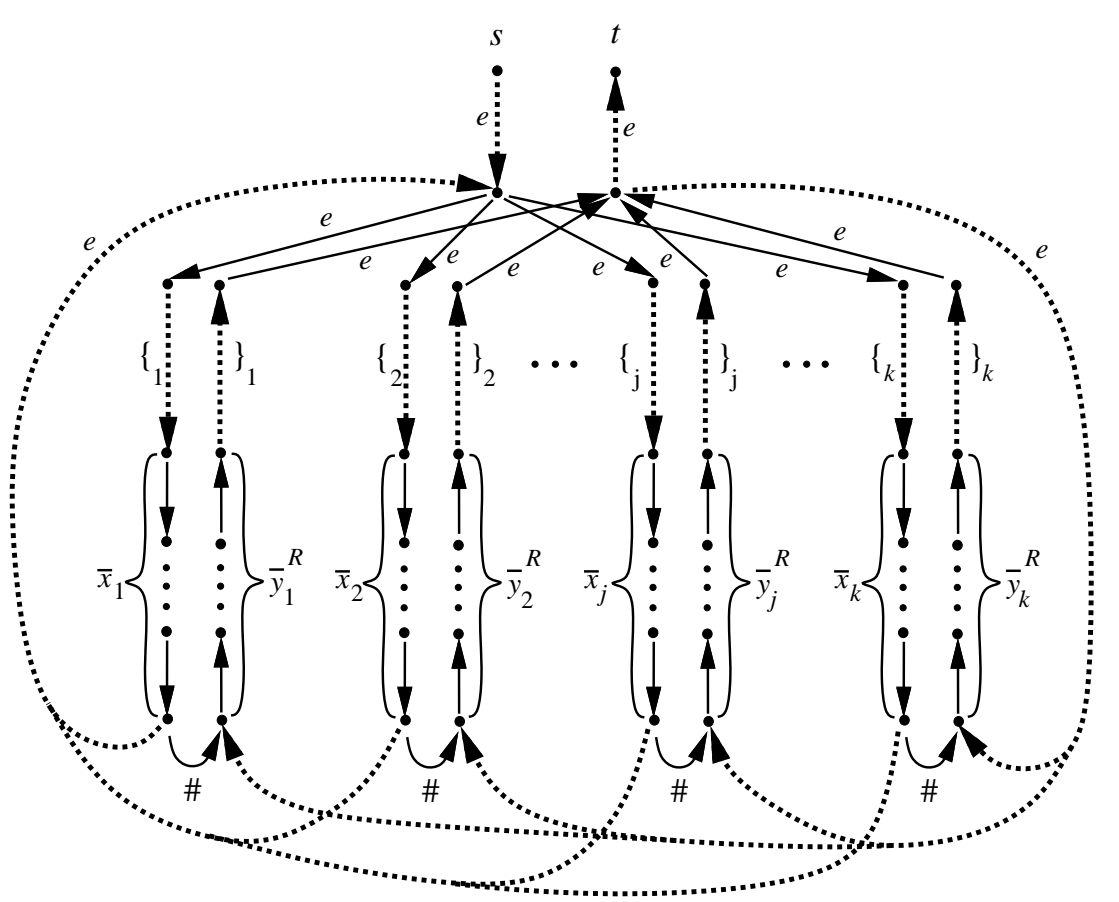

Figure 1. A schematic diagram of the graph that would be constructed for an instance of P-PCP $\bar{X}=\bar{x}_{1}, \bar{x}_{2}, \cdots, \bar{x}_{j}, \cdots, \bar{x}_{k} ; \bar{Y}^{R}=\bar{y}_{1}^{R}, \bar{y}_{2}^{R}, \cdots, \bar{y}_{j}^{R}, \cdots, \bar{y}_{k}^{R}$. 
sequence " $\}_{j} e$ ". The dotted edges labeled $e$ around the outsides of Fig. 1 serve to connect each $\bar{x}$-string segment to all of the other $\bar{x}$-string segments, and each $\bar{y}^{R}$-string segment to all of the other $\bar{y}^{R}$-string segments. Any number of $\bar{x}$-string segments can be concatenated together to form a path in any order; however, each such segment is labeled in the word of the path by the appropriate " $\{$ "symbol. Similarly, any number of $\bar{y}^{R}$-string segments can be concatenated together to form a path in any order; however, each such segment is labeled in the word of the path by the appropriate "\}" symbol.

The fact that certain edges in Fig. 1 are dotted has no special significance; they are displayed in this way to highlight the fact that these edges correspond to interprocedural data-dependence edges in dependence graphs. This correspondence will be made clear in Section 4 (cf. Fig. 4).

By following one of the edges that is labeled with "\#”, a path can pass from an $\bar{x}$-string segment to a $\bar{y}^{R}$-string segment. However, once a \#-edge is taken, the path can only be extended with $\bar{y}^{R}$-string segments. Consequently, all paths from $s$ to $t$ are of the form:

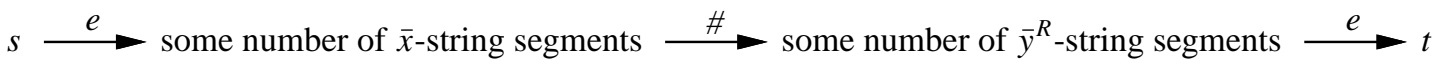

Here we see the reason for the remark made in footnote 6: In the word of a path from $s$ to $t$, each occurrence of " $\{$ " is immediately preceded by two occurrences of the symbol $e$, and each occurrence of " $\}$ " is immediately followed by two occurrences of $e$. Technically, the definition of a (candidate) P-PCP solution

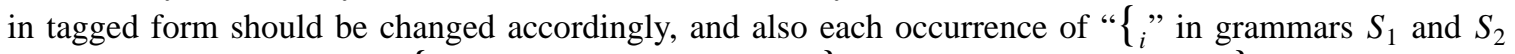
should be replaced with "e $e\left\{{ }_{i} \text { ", and each occurrence of " }\right\}_{i}$ " should be replaced with " $\}_{i} e e$ ".

We now observe that

- If there is an $L\left(S_{1}\right) \cap L\left(S_{2}\right)$-path $P$ from $s$ to $t$, a solution to the instance of P-PCP can be read off from the word of $P$ by reading it as a P-PCP solution in tagged form.

- If the instance of P-PCP has the solution $i_{1}, i_{2}, \cdots, i_{j}, \cdots, i_{m}$, then we can find an $L\left(S_{1}\right) \cap L\left(S_{2}\right)$-path $P$ from $s$ to $t$ by

(i) following the $e$ edge from $s$,

(ii) choosing $\bar{x}$-string segments in the order $\bar{x}_{i_{1}}, \bar{x}_{i_{2}}, \cdots, \bar{x}_{i_{j}}, \cdots, \bar{x}_{i_{m}}$, thereby generating a subpath whose word is $e\left\{i_{i_{1}} \bar{x}_{i_{1}} e e\left\{i_{i_{2}} \bar{x}_{i_{2}} \cdots e e\left\{\left\{_{i_{j}} \bar{x}_{i_{j}} \cdots e e\left\{i_{i_{m}} \bar{x}_{i_{m}}\right.\right.\right.\right.\right.$,

(iii) following the \#-edge from the $i_{m}^{\text {th }} \bar{x}$-string segment to the $i_{m}^{\text {th }} \bar{y}^{R}$-string segment,

(iv) choosing $\bar{y}^{R}$-string segments in the order $\bar{y}_{i_{m}}^{R}, \cdots, \bar{y}_{i_{j}}^{R}, \cdots, \bar{y}_{i_{2}}^{R}, \bar{y}_{i_{1}}^{R}$, thereby generating a subpath whose word is $\left.\bar{y}_{i_{m}}^{R}\right\}_{i_{m}}$ e e $\left.\cdots \bar{y}_{i_{j}}^{R}\right\}_{i_{j}}$ e e $\left.\cdots \bar{y}_{i_{2}}^{R}\right\}_{i_{2}}$ e e $\left.\bar{y}_{i_{1}}^{R}\right\}_{i_{1}}$ e,

(v) following the $e$ edge to $t$.

The word of this path exhibits the solution to the instance of P-PCP in tagged form (modulo the extra occurrences of $e$ ). As we observed earlier, $L\left(S_{1}\right) \cap L\left(S_{2}\right)$ consists of exactly the solutions, in tagged form, to the given instance of P-PCP. Hence, the path constructed above is an $L\left(S_{1}\right) \cap L\left(S_{2}\right)$-path from $s$ to $t$.

We shall call a graph constructed in the manner described above a $P$-PCP graph. For the particular case of the instance of P-PCP introduced in Example 2.4, the corresponding P-PCP graph is shown in Fig. 2.

The above observations prove the following lemma:

LEMMA 3.1. Given an instance of P-PCP (with corresponding grammars $S_{1}$ and $S_{2}$, and P-PCP graph $G)$, there is an $L\left(S_{1}\right) \cap L\left(S_{2}\right)$-path from s to $t$ in $G$ if and only if the instance of P-PCP has a solution.

\section{UNDECIDABILITY OF CONTEXT-SENSITIVE, STRUCTURE-TRANSMITTED DATA-DEPEN- DENCE ANALYSIS}

In this section, we show how a slight modification of the construction presented in the previous section implies that it would be impossible to create a precise algorithm for context-sensitive, structure-transmitted data-dependence analysis. In particular, we construct a family of programs whose data-dependence graphs encode the P-PCP graphs. 


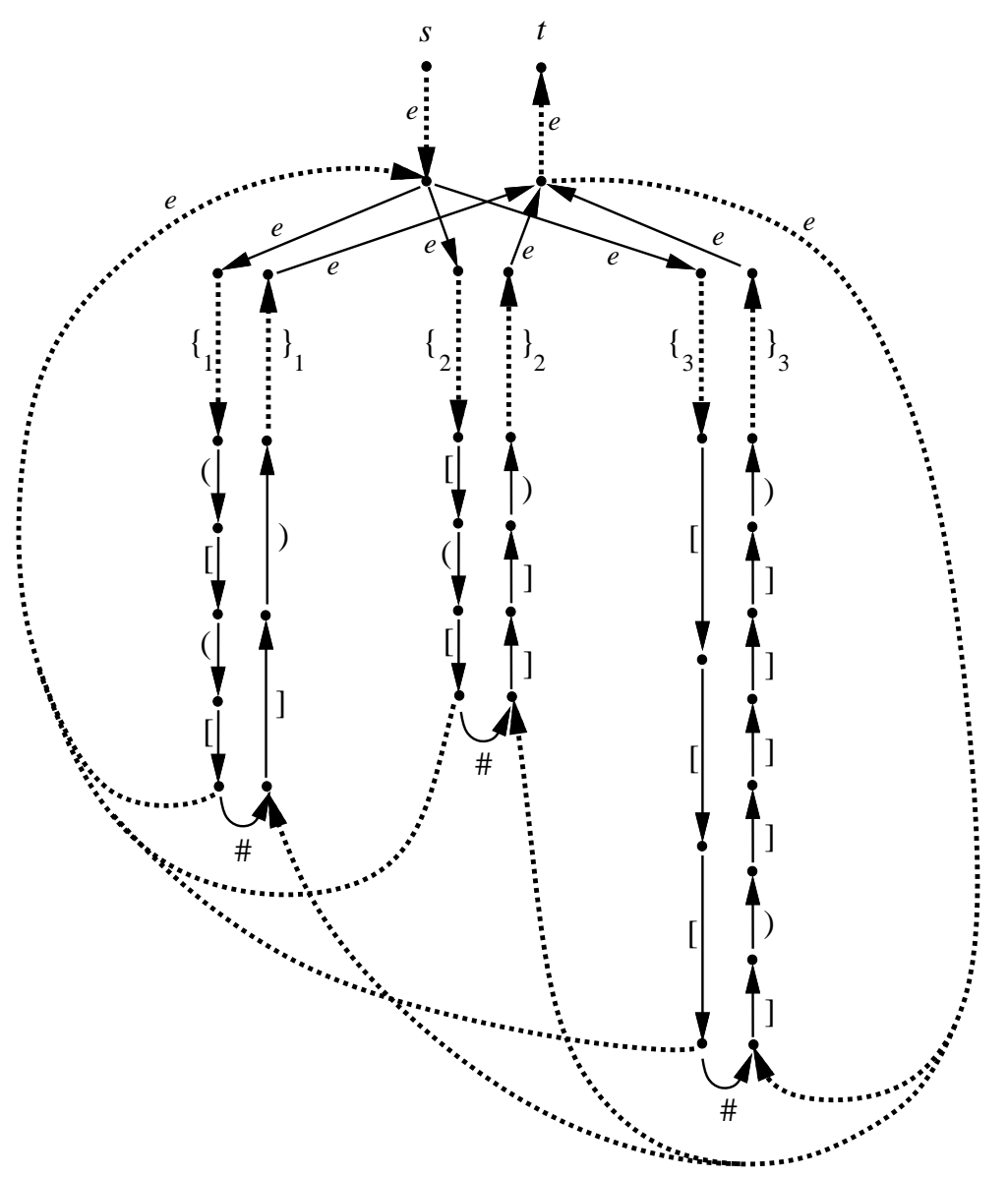

Figure 2. The P-PCP graph that would be constructed for the instance of P-PCP given in Example 2.4. This graph contains an $L\left(S_{1}\right) \cap L\left(S_{2}\right)$-path from $s$ to $t$ whose word is

$$
e e\left\{_{1}\left(\left[\left(\left[e e\left\{_{2}\left[\left(\left[e e\left\{_{3}\left[\left[\left[e e\left\{_{1}\left([([\#])\}_{1} e e\right]\right)\right]\right]\right]\right]\right)\right\}_{3} e e\right]\right]\right)\right\}_{2} e e\right]\right)\right\}_{1} e e,
$$

which indicates that the instance of P-PCP given in Example 2.4 has the solution 1, 2, 3, 1.

For instance, Fig. 3 shows a $\mathrm{C}$ program fragment whose data-dependence graph (see Fig. 4) corresponds to the instance of P-PCP given in Example 2.4. A context-sensitive, structure-transmitted data-dependence analysis should report that variable $x$ may have the value atom("A") at program point $t$ (which corresponds to the fact that this instance of P-PCP has the solution 1, 2, 3, 1). In Fig. 3, the symbols " $\{$ " and " $\}_{j}$ " correspond to data dependences associated with the call from procedure $f$ to procedure $f j$ and the return from $f j$ to $f$, respectively; the symbol "(" corresponds to a data construction in which the value is placed in the first position of a cons; the symbol "[" corresponds to a data construction in which the value is placed in the second position of a cons; the symbol ")" corresponds to a selection via car; the symbol "]" corresponds to a selection via cdr. Data dependences associated with calls to procedure $f$ are labeled by symbols of the form " $<_{i}$ "; data dependences associated with corresponding returns from $\mathrm{f}$ are labeled by " $\rangle_{i}$ "; the symbol "\#" corresponds to a data dependence that occurs when a (recursive) call on $\mathrm{f}$ is finally 
bypassed. $^{7}$

When inspecting Fig. 3, the reader should keep in mind that the left-to-right encoding of a string-where a string consists of either all open parentheses or all closed parentheses_corresponds to working one's way out from the inner occurrence of $\mathrm{x}$ in the expression that encodes the string.

The idea illustrated in Figs. 3 and 4 carries over to all instances of P-PCP: In general, the pair of strings $\bar{x}_{j}, \bar{y}_{j}^{R}$ is encoded by a procedure of the form

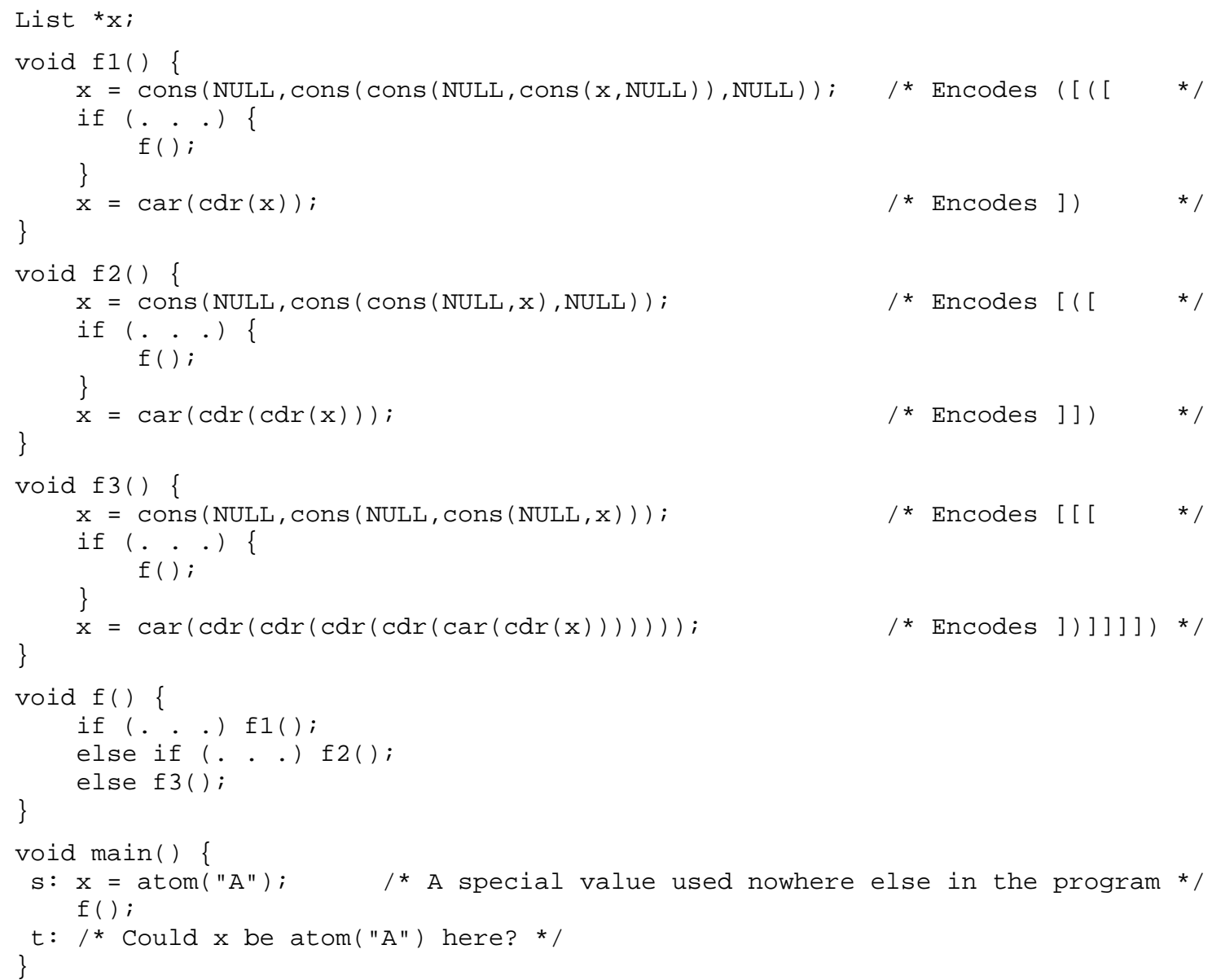

Figure 3. The $\mathrm{C}$ program scheme that would be constructed for the instance of P-PCP given in Example 2.4. The relevant part of this program's data-dependence graph is shown in Fig. 4.

${ }^{7}$ The role of the labels "〈" and "〉" is similar to that of " $\{$ " and " $\}$ ", respectively, in that both kinds of parenthesis pairs encode procedure call/return. However, $\langle$ and $\rangle$ have been introduced as separate symbols to emphasize the fact that the calls to procedure $f$ play a different role in the construction than the calls to the $\mathrm{f} j$ procedures. 


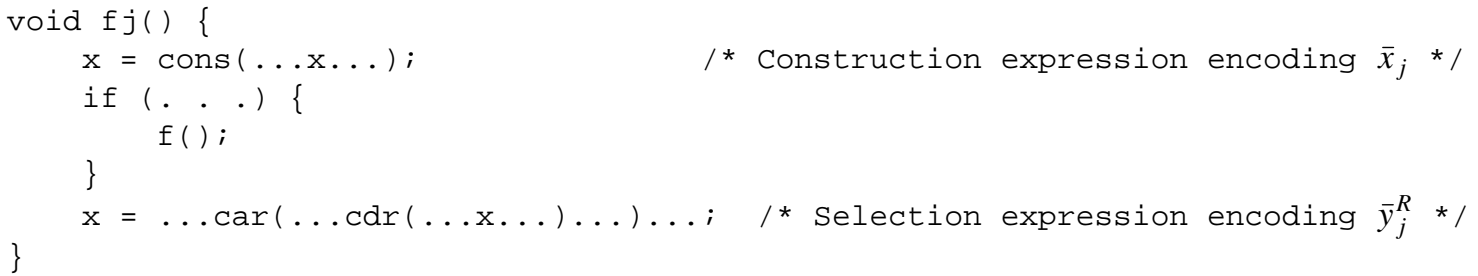

Procedure $f$ has the form

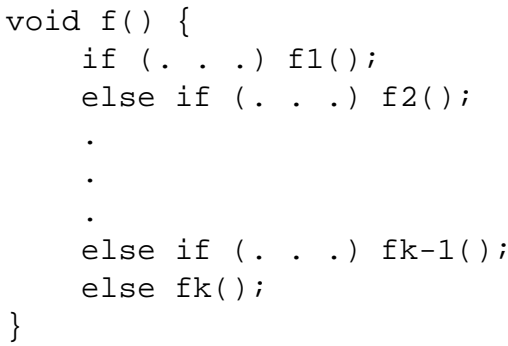

Procedure main is the same as in Fig. 3.

The undecidability of context-sensitive, structure-transmitted data-dependence analysis follows from two properties: (i) the data-dependence graph for a program of the form given above is very much like the PPCP graph for the given instance of P-PCP (see Fig. 1), and (ii) variable $x$ can have the value atom ("A") at program point $t$ if and only if there is a path from $s$ to $t$ whose word is in a language very similar to $L\left(S_{1}\right) \cap L\left(S_{2}\right)$ (except that 〈's and 〉's must also be balanced).

For instance, the portion of the data-dependence graph shown in Fig. 4 (for the program given in Fig. 3) is identical to the P-PCP graph shown in Fig. 2, except that certain dotted edges, corresponding to calls to $f$ and returns from $\mathrm{f}$, now have labels of the form $\zeta_{i}$ or $\rangle_{i}{ }^{8}$ Therefore, the path language of interest for identifying context-sensitive, structure-transmitted data dependences must now incorporate the labels $\left\langle_{i} \text { and }\right\rangle_{i}$ (for $0 \leq i \leq k$ ). Formally, this is accomplished by considering $L\left(S_{1}{ }^{\prime}\right) \cap L\left(S_{2}{ }^{\prime}\right)$-paths, where the grammars $S_{1}{ }^{\prime}$ and $S_{2}{ }^{\prime}$ are defined as follows:

$$
\begin{aligned}
& S_{1}{ }^{\prime} \rightarrow e\left\langle{ }_{0} S_{1}{ }^{\prime \prime}\right\rangle_{0} e \\
& S_{1}{ }^{\prime \prime} \rightarrow\left(S_{1}{ }^{\prime \prime}\right) \\
& \text { | [ } S_{1}^{\prime \prime} \text { ] } \\
& \text { | (\#) } \\
& \text { [ [ \# ] } \\
& \text { | } e\left\{{ }_{i} S_{1}{ }^{\prime \prime} \quad \text { for } 1 \leq i \leq k\right. \\
& \text { | }\left\langle_{i} S_{1}^{\prime \prime} \quad \text { for } 1 \leq i \leq k\right. \\
& \text { | } \left.S_{1}{ }^{\prime \prime}\right\}_{i} e \quad \text { for } 1 \leq i \leq k \\
& \left.S_{1}^{\prime \prime}\right\rangle_{i} \quad \text { for } 1 \leq i \leq k \\
& S_{2}{ }^{\prime} \rightarrow e\left\langle S_{0} S_{2}{ }^{\prime \prime}\right\rangle_{0} e \\
& S_{2}{ }^{\prime \prime} \rightarrow e\left\{{ }_{i} \bar{x}_{i}\left\langle S_{i} S_{2}{ }^{\prime \prime}\right\rangle_{i} \bar{y}_{i}^{R}\right\}_{i} e \quad \text { for } 1 \leq i \leq k \\
& \text { I } e\left\{{ }_{i} \bar{x}_{i} \# \bar{y}_{i}^{R}\right\}_{i} e \quad \text { for } 1 \leq i \leq k
\end{aligned}
$$

We also change the notion of a P-PCP solution in tagged form to one in which each occurrence of $\bar{x}_{i}$ (except for the innermost one) is immediately followed by an occurrence of $\left\langle_{i}\right.$, and each occurrence of $\bar{y}_{i}^{R}$ (except for the innermost one) is immediately preceded by an occurrence of $\rangle_{i}$.

${ }^{8}$ The only elements omitted from the data-dependence graph shown in Fig. 4 concern dependences on NULL. These are irrelevant to the question of how atom ("A") flows through the program—and to our embedding of P-PCP graphs in data-dependence graphs. 


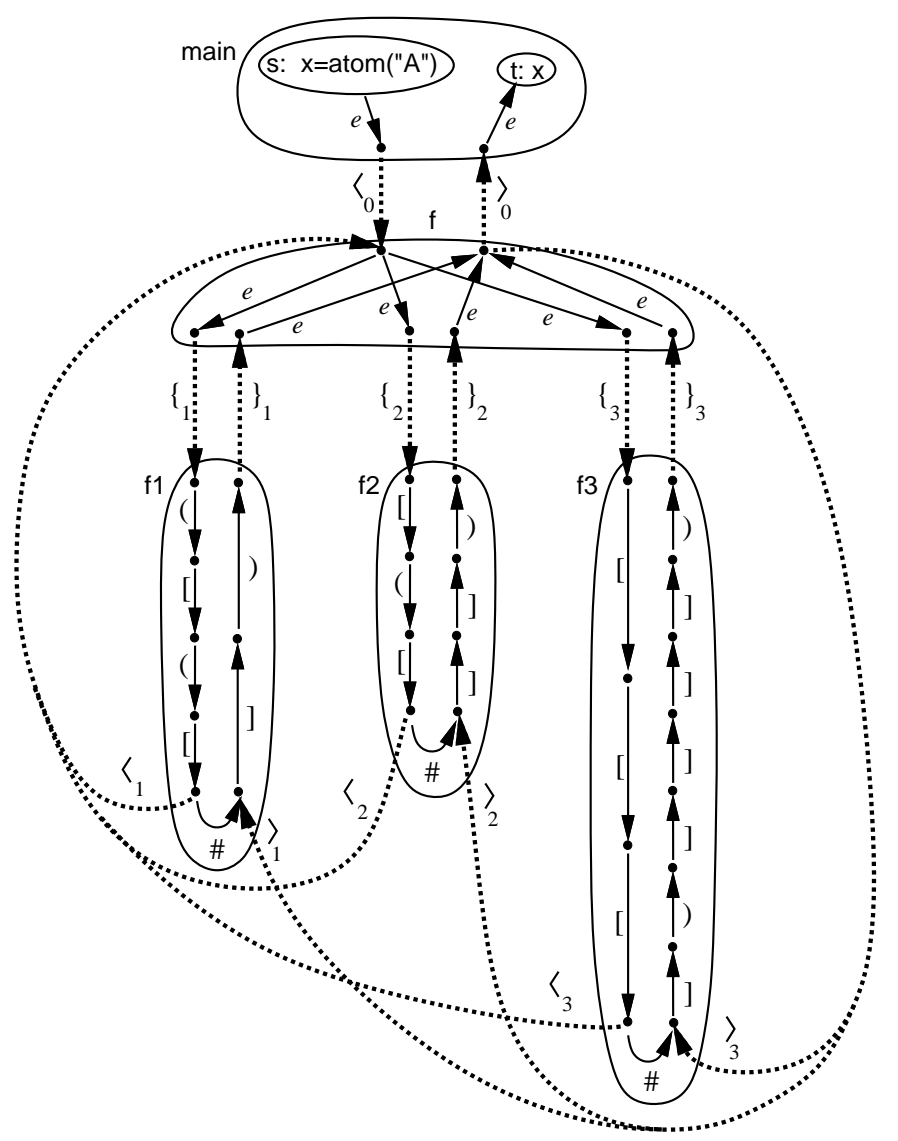

Figure 4. Relevant parts of the data-dependence graph for the program shown in Fig. 3. This corresponds to the PPCP graph shown in Fig. 2, except that certain dotted edges now have labels of the form $\left\langle{ }_{i} \text { or }\right\rangle_{i}$.

By the same argument used to prove Lemma 3.1, there is an $L\left(S_{1}{ }^{\prime}\right) \cap L\left(S_{2}{ }^{\prime}\right)$-path from s to $t$ in the datadependence graph if and only if the given instance of P-PCP has a solution. Therefore, a context-sensitive, structure-transmitted data-dependence analysis would determine that $\mathrm{x}$ could have the value atom ("A ") at program point $t$ if and only if the given instance of P-PCP has a solution. Consequently, context-sensitive, structure-transmitted data-dependence analysis is undecidable (i.e., an algorithm for context-sensitive, structure-transmitted data-dependence analysis cannot exist).

\section{CONCLUSIONS AND IMPLICATIONS FOR OTHER PROGRAM-ANALYSIS FRAMEWORKS}

Earlier work by the author and his colleagues has demonstrated the usefulness of formulating programanalysis problems in terms of graph-reachability questions [32]. This approach has been used to obtain a number of positive results about program-analysis problems (specifically, polynomial-time algorithms for solving a variety of different problems $[15,28,30,16,29,34,25])$. The present paper demonstrates that this viewpoint is also valuable from the standpoint of obtaining negative results about program-analysis problems (see also [31]).

The undecidability result in this paper concerns a situation in which there are two interleaved patterns of matching "events". Viewed more broadly, the notions of "interleaved matched-parenthesis paths" and "PPCP solutions in tagged form" are two concepts that can provide insight into whether other program-analysis problems are undecidable. For instance, Ramalingam showed recently that synchronization-sensitive, context-sensitive interprocedural analysis of multi-tasking concurrent programs is undecidable [27]. His 
result was inspired by the one given in the present paper, using the insight that synchronization-sensitive, context-sensitive interprocedural analysis also involves two interleaved patterns of matching events.

\section{Set Constraints and Set-Based Analysis}

Following earlier work by Reynolds [33] and Jones and Muchnick [18], a number of people in recent years have explored the use of set constraints for analyzing programs. Set constraints are typically used to collect a superset of the set of values that the program's variables may hold during execution. Typically, a set variable is created for each program variable at each program point; set constraints are generated that approximate the program's behavior; program analysis then becomes a problem of finding the least solution of the set-constraint problem. Set constraints have been used both for program analysis $[33,18,1,11,12]$, and for type inference [2,3].

Melski and Reps have obtained a number of results on the relationship between certain classes of set constraints and CFL-reachability [25]. Their results establish relationships in both directions: They showed that CFL-reachability problems and a subclass of what have been called definite set constraints [10] are equivalent. That is, given a CFL-reachability problem, it is possible to construct a set-constraint problem whose answer gives the solution to the CFL-reachability problem; likewise, given a set-constraint problem, it is possible to construct a CFL-reachability problem whose answer gives the solution to the set-constraint problem. It is also shown in [25] that CFL-reachability is equivalent to a class of set constraints that was designed to be useful for (context-insensitive) analysis of programs written in a higher-order language-socalled "set-based analysis" [11]. The results of Sections 3 and 4 imply that if you start with a version of context-insensitive set-based analysis that is at least as precise as the context-insensitive structure-transmitted data-dependence analysis illustrated in Example 1.5, then it is impossible to create an algorithm for the context-sensitive version of your set-based analysis, even for a first-order language.

\section{Control-Flow Analysis}

In [35], Sharir and Pnueli defined two methods for carrying out interprocedural dataflow analysis so as to ensure that the propagation of dataflow information respects the fact that when a procedure finishes it returns to the site of the most recent call. In one of their methods, the so-called "call-strings approach", each piece of dataflow information is tagged with a call string that records the history of uncompleted procedure calls along which that data has propagated. The call string on a piece of information is updated whenever a propagation step associated with a call statement or return statement is performed. The information that would be obtained, in principle, if call strings were allowed to grow arbitrarily long is called the call-strings- $\infty$ solution.

Sharir and Pnueli show that, for distributive dataflow-analysis problems over a finite semilattice, it is possible to restrict the length of call strings to some fixed length (where the bound on the length required is quadratic in the size of the lattice and linear in the number of call sites in the program) and yet still obtain a result that is equivalent in precision to the call-strings- $\infty$ solution. By suitable means, approximate (but safe) solutions can also be obtained using shorter call strings; limiting call strings to length $k$ defines the call-strings- $k$ solution.

In considering algorithms for interprocedural dataflow analysis, one should be careful not to confuse two separate issues:

(i) Whether an algorithm computes a solution equal in precision to the call-strings- $\infty$ solution.

(ii) Whether an algorithm computes its solution by actually tracking entities labeled by call strings (e.g., of some length $k$ ).

A type-(ii) algorithm typically has worst-case running time that is exponential in $k$. However, for suitably restricted classes of interprocedural dataflow-analysis problems, there are algorithms with property (i), yet 
their worst-case running times are polynomial in the size of the program $;{ }^{9}$ these algorithms use dynamic programming, rather than utilizing entities labeled with explicit call strings [30,34]. For the same class of problems, a type-(ii) algorithm will, in general, have exponential running time.

These results provide an interesting contrast with those that have been obtained on a program-analysis problem of interest to the functional-programming community: the problem of " $k$-CFA", or "control-flow analysis" (for higher-order programming languages) using call strings of length $k[36,17,13,26]$. The goal of control-flow analysis is to track data and control flow in the presence of first-class (anonymous) functions, data constructors, and selectors. Many of the algorithms that have been given for $k$-CFA are type-(ii) algorithms (in the sense mentioned above), in that they actually track entities labeled by call strings of length $\leq k$. In general, the running time of these algorithms is exponential in $k$.

Similar to the concept of the call-strings- $\infty$ solution to an interprocedural dataflow-analysis problem, the $\infty$-CFA solution is what would be obtained, in principle, if call strings were allowed to grow arbitrarily long. The results of Sections 3 and 4 imply that, in general, when data constructors and selectors are to be taken into account, $\infty$-CFA is undecidable. That is, in the presence of data constructors and selectors, the $\infty$-CFA solution cannot be computed.

\section{ACKNOWLEDGEMENTS}

I am grateful for discussions that I had about the problem with F. Nielson, G. Ramalingam, S. Horwitz, and D. Melski.

\section{REFERENCES}

1. Aiken, A. and Murphy, B.R., "Implementing regular tree expressions," pp. 427-447 in Func. Prog. and Comp. Arch., Fifth ACM Conf., (Cambridge, MA, Aug. 26-30, 1991), Lec. Notes in Comp. Sci., Vol. 523, ed. J. Hughes, Springer-Verlag, New York, NY (1991).

2. Aiken, A. and Murphy, B.R., "Static type inference in a dynamically typed language," pp. 279-290 in Conf. Rec. of the Eighteenth ACM Symp. on Princ. of Prog. Lang., (Orlando, FL, Jan. 1991), ACM, New York, NY (1991).

3. Aiken, A. and Wimmers, E.L., "Type inclusion constraints and type inference," pp. 31-41 in Sixth Conf. on Func. Prog. and Comp. Arch., (Copenhagen, Denmark), (June 1993).

4. Callahan, D., "The program summary graph and flow-sensitive interprocedural data flow analysis," Proc. of the ACM SIGPLAN 88 Conf. on Prog. Lang. Design and Implementation, (Atlanta, GA, June 22-24, 1988), SIGPLAN Not. 23(7) pp. 47-56 (July 1988).

5. Cooper, K.D. and Kennedy, K., "Interprocedural side-effect analysis in linear time," Proc. of the ACM SIGPLAN 88 Conf. on Prog. Lang. Design and Implementation, (Atlanta, GA, June 22-24, 1988), SIGPLAN Not. 23(7) pp. 57-66 (July 1988).

6. Cooper, K.D. and Kennedy, K., "Fast interprocedural alias analysis," pp. 49-59 in Conf. Rec. of the Sixteenth ACM Symp. on Princ. of Prog. Lang., (Austin, TX, Jan. 11-13, 1989), ACM, New York, NY (1989).

7. Emami, M., Ghiya, R., and Hendren, L.J., "Context-sensitive interprocedural points-to analysis in the presence of function pointers," Proc. of the ACM SIGPLAN 94 Conf. on Prog. Lang. Design and Implementation, (Orlando, FL, June 22-24, 1994), SIGPLAN Not. 29(6) pp. 242-256 (June 1994).

8. Harrison, M.A., Introduction to Formal Language Theory, Addison-Wesley, Reading, MA (1978).

9. Hecht, M.S., Flow Analysis of Computer Programs, North-Holland, New York, NY (1977).

${ }^{9}$ In their Theorem 7-3.4, Sharir and Pnueli establish that the greatest fixed point of a certain set of equations equals the meet-over-allvalid-paths (MOVP) solution. In their Theorem 7-4.6, they establish that the MOVP solution equals the call-strings- $\infty$ solution. As for algorithms, Sharir and Pnueli give a worklist algorithm for finding the greatest fixed point of the set of equations. However, Section 7-3 of [35] presents a fairly general framework: in particular, the only assumption about the semilattice is that it is finite. Because of the way their dynamic-programming algorithm tabulates information, when the size of the semilattice is exponential in the size of the program, the algorithm may use time exponential in the size of the program.

To achieve a polynomial time bound, the tricks are:

- To restrict attention to a certain class of semilattices. However, this class can include semilattices whose size is exponential in the size of the program (e.g., the powerset of the program points, the powerset of the program's variables, etc.).

- To tabulate the Sharir-Pnueli $\phi$ functions pointwise (e.g., on singletons, rather than entire sets).

This is essentially what is done in [30] for the class of finite-distributive-subset problems, and in [34] for the larger class of distributive environment problems. 
10. Heintze, N. and Jaffar, J., "A decision procedure for a class of set constraints," Tech. Rep. CMU-CS-91-110, School of Computer Science, Carnegie Mellon Univ., Pittsburgh, PA (1991).

11. Heintze, N., "Set-based analysis of ML programs," Tech. Rep. CMU-CS-93-193, School of Computer Science, Carnegie Mellon Univ., Pittsburgh, PA (July 1993).

12. Heintze, N. and Jaffar, J., "Set constraints and set-based analysis," in 2nd Workshop on Principles and Practice of Constraint Programming, (May 1994).

13. Heintze, N. and McAllester, D., "Linear-time subtransitive control flow analysis," Proc. of the ACM SIGPLAN 97 Conf. on Prog. Lang. Design and Implementation, (Las Vegas, Nevada, June 15-18, 1997), SIGPLAN Not. 32(5) pp. 261-272 (May 1997).

14. Hopcroft, J.E. and Ullman, J.D., Introduction to Automata Theory, Languages, and Computation, Addison-Wesley, Reading, MA (1979).

15. Horwitz, S., Reps, T., and Binkley, D., "Interprocedural slicing using dependence graphs," ACM Trans. Program. Lang. Syst. 12(1) pp. 26-60 (Jan. 1990).

16. Horwitz, S., Reps, T., and Sagiv, M., "Demand interprocedural dataflow analysis," SIGSOFT 95: Proc. of the Third ACM SIGSOFT Symp. on the Found. of Softw. Eng., (Wash., DC, Oct. 10-13, 1995), ACM SIGSOFT Softw. Eng. Notes 20(4) pp. 104-115 (1995).

17. Jagannathan, S. and Weeks, S., "A unified treatment of flow analysis in higher-order languages," pp. 393-406 in Conf. Rec. of the Twenty-Second ACM Symp. on Princ. of Prog. Lang., (San Francisco, CA, Jan. 23-25, 1995), ACM, New York, NY (1995).

18. Jones, N.D. and Muchnick, S.S., "Flow analysis and optimization of Lisp-like structures," pp. 102-131 in Program Flow Analysis: Theory and Applications, ed. S.S. Muchnick and N.D. Jones, Prentice-Hall, Englewood Cliffs, NJ (1981).

19. Khedker, U.P. and Dhamdhere, D.M., "A generalized theory of bit vector data flow analysis," ACM Trans. Program. Lang. Syst. 16(5) pp. 1472-1511 (Sept. 1994).

20. Kou, L.T., "On live-dead analysis for global data flow problems," J. ACM 24(3) pp. 473-483 (July 1977).

21. Kuck, D.J., The Structure of Computers and Computations, Vol. 1, John Wiley \& Sons, New York, NY (1978).

22. Kuck, D.J., Kuhn, R.H., Leasure, B., Padua, D.A., and Wolfe, M., "Dependence graphs and compiler optimizations," pp. 207-218 in Conf. Rec. of the Eighth ACM Symp. on Princ. of Prog. Lang., (Williamsburg, VA, Jan. 26-28, 1981), ACM, New York, NY (1981).

23. Lewis, H.R. and Papadimitriou, C.H., Elements of the Theory of Computation, Prentice-Hall, Englewood Cliffs, NJ (1981).

24. McCarthy, J., "A basis for a mathematical theory of computation," pp. 33-70 in Computer Programming and Formal Systems, ed. Braffort and Hershberg, North-Holland, Amsterdam (1963).

25. Melski, D. and Reps, T., "Interconvertibility of a class of set constraints and context-free language reachability," Theor. Comp. Sci. 248(1-2)(Nov. 2000). (To appear.)

26. Nielson, F. and Nielson, H.R., "Infinitary control flow analysis: A collecting semantics for closure analysis," pp. 332-345 in Conf. Rec. of the Twenty-Fourth ACM Symp. on Princ. of Prog. Lang., (Paris, France, Jan. 15-17, 1997), ACM, New York, NY (1997).

27. Ramalingam, G., "Context-sensitive synchronization-sensitive analysis is undecidable," Res. Rep. RC 21493, IBM T.J. Watson Res. Cent., Yorktown Heights, NY (May 1999).

28. Reps, T., Horwitz, S., Sagiv, M., and Rosay, G., "Speeding up slicing," SIGSOFT 94: Proc. of the Second ACM SIGSOFT Symp. on the Found. of Softw. Eng., (New Orleans, LA, Dec. 7-9, 1994), ACM SIGSOFT Softw. Eng. Notes 19(5) pp. 11-20 (Dec. 1994).

29. Reps, T., "Shape analysis as a generalized path problem," pp. 1-11 in Proc. of the ACM SIGPLAN Symp. on Part. Eval. and Sem.-Based Prog. Manip. (PEPM 95), (La Jolla, California, June 21-23, 1995), ACM, New York, NY (1995).

30. Reps, T., Horwitz, S., and Sagiv, M., "Precise interprocedural dataflow analysis via graph reachability," pp. 49-61 in Conf. Rec. of the Twenty-Second ACM Symp. on Princ. of Prog. Lang., (San Francisco, CA, Jan. 23-25, 1995), ACM, New York, NY (1995).

31. Reps, T., "On the sequential nature of interprocedural program-analysis problems," Acta Inf. 33 pp. $739-757$ (1996).

32. Reps, T., "Program analysis via graph reachability," Information and Software Technology 40(11-12) pp. 701-726 Elsevier Science, (Nov./Dec. 1998).

33. Reynolds, J.C., "Automatic computation of data set definitions," pp. 456-461 in Information Processing 68: Proc. of the IFIP Congress 68, North-Holland, New York, NY (1968).

34. Sagiv, M., Reps, T., and Horwitz, S., "Precise interprocedural dataflow analysis with applications to constant propagation," Theor. Comp. Sci. 167 pp. 131-170 (1996).

35. Sharir, M. and Pnueli, A., "Two approaches to interprocedural data flow analysis," pp. 189-233 in Program Flow Analysis: Theory and Applications, ed. S.S. Muchnick and N.D. Jones, Prentice-Hall, Englewood Cliffs, NJ (1981).

36. Shivers, O., "Control flow analysis in Scheme," Proc. of the ACM SIGPLAN 88 Conf. on Prog. Lang. Design and Implementation, (Atlanta, GA, June 22-24, 1988), SIGPLAN Not. 23(7) pp. 164-174 (July 1988).

37. Wilson, R.P. and Lam, M.S., "Efficient context-sensitive pointer analysis for C programs," Proc. of the ACM SIGPLAN 95 Conf. on Prog. Lang. Design and Implementation, (La Jolla, CA, June 18-21, 1995), SIGPLAN Not. 30(6) pp. 1-12 (June 1995).

38. Yannakakis, M., "Graph-theoretic methods in database theory," pp. 230-242 in Proc. of the Ninth ACM Symp. on Princ. of Database Syst., (1990). 